TRABAJO ORIGINAL

\title{
Elevación del índice neutrófilo/linfocito y su relación con la proteína $C$ reactiva en pacientes con diabetes mellitus tipo 2
}

\author{
Elevation of the neutrophil/lymphocyte ratio and its relationship \\ to C-reactive protein in patients with type 2 diabetes mellitus
}

Adriana Pedreáñez ${ }^{1}$, Jesús Mosquera-Sulbarán², Jorge Robalino³, Diego Tene ${ }^{4}$, Nelson Muñóz ${ }^{5}$

\section{RESUMEN}

Introducción: la proporción entre el recuento absoluto de neutrófilos y el recuento absoluto de linfocitos (índice de neutrófilos/linfocitos, INL) se ha convertido en los últimos años en un marcador crucial de inflamación sistémica, y se ha descrito que su elevación se relaciona con numerosas enfermedades inflamatorias crónicas.

Objetivos: determinar el índice de neutrófilos/linfocitos (INL) en pacientes con diabetes mellitus tipo 2 (DM2), comparar con no diabéticos y establecer su correlación con la concentración de proteína $C$ reactiva ultrasensible en una población de la localidad de Riobamba, Ecuador.

Materiales y métodos: se realizó una investigación de tipo descriptiva, correlacional, de corte transversal, en el período comprendido desde julio de 2019 a febrero de 2020. Se seleccionaron 80 individuos para participar en el proyecto: 25 sujetos controles y 55 pacientes con diagnóstico de DM2. A cada sujeto se le extrajo una muestra de sangre en ayunas para la determinación de glucosa, colesterol total, triglicéridos, HDL colesterol, LDL colesterol, proteína $C$ reactiva ultrasensible (PCR-us), hemoglobina glicosilada ( $\mathrm{HbA} 1 \mathrm{c})$, recuento total de leucocitos, neutrófilos y linfocitos.

Resultados: se encontró un incremento significativo en la concentración de glucosa $(p<0,0001), \mathrm{HbA} 1 \mathrm{c}(\mathrm{p}<0,0001)$, índice de masa corporal (IMC) $(p<0,0001)$, PCR-us $(p<0,0001)$, recuento absoluto de neutrófilos $(p=0,001)$, recuento absoluto de linfocitos $(p=0,04)$ e INL $(p=0,0005)$, y una reducción significativa del HDL colesterol $(p=0,02)$ en los pacientes con DM2 vs los controles. Se observó una correlación positiva $(p<0,0001 ; r=0,7774)$ entre el INL y la PCR-us en los pacientes con DM2.

Conclusiones: los pacientes con DM2 experimentaron elevación en el INL que se correlacionó con el incremento en la concentración de la PCR-us.

Palabras clave: diabetes; inflamación; índice neutrófilo/linfocito.

Revista de la Sociedad Argentina de Diabetes 2021; Vol. 55 (77- 83)

\section{ABSTRACT}

Introduction: the ratio between the absolute neutrophil count and the absolute lymphocyte count (neutrophil/lymphocyte ratio, NLR) has become a crucial marker of systemic inflammation in recent years, and its elevation has been described as being related to numerous chronic inflammatory diseases. Objectives: to determine the neutrophil/lymphocyte ratio (NLR) in patients with type 2 diabetes mellitus (T2DM), to compare with non-diabetics and to establish its correlation with the concentration of ultrasensitive $C$-reactive protein in a population of the town of Riobamba, Ecuador.

Materials and methods: a descriptive, correlational, crosssectional, research was conducted from July 2019 to February 2020. Eighty individuals were selected to participate in the project, 25 control subjects and 55 patients with a diagnosis of T2DM. Each subject had a fasting blood sample drawn for the determination of glucose, total cholesterol, triglycerides, HDL cholesterol, LDL cholesterol, ultrasensitive C-reactive protein (hs-CRP), glycosylated hemoglobin (HbA1c), total leukocyte count, neutrophils and lymphocytes.

Results: a significant increase in glucose concentration $(p<0.0001)$, HbA1c $(p<0.0001)$, body mass index (BMI) $(p<0.0001)$, hs-CRP $(p<0.0001)$, absolute neutrophil count $(p=0.001)$, absolute lymphocyte count $(p=0.04)$, and NLR $(p=0.0005)$, and a significant reduction in HDL cholesterol $(p=0.02)$, were found in patients with T2DM vs controls. A positive correlation $(p<0.0001 ; r=0.7774)$ was observed between NLR and hs-CRP in patients with T2DM.

Conclusions: patients with T2DM experience elevation in NLR which correlates with increase in hs-CRP concentration.

Key words: diabetes; inflammation; neutrophil/lymphocyte ratio.
PhD en Inmunología, Jefe de la Cátedra de Inmunología, Escuela de Bioanálisis, Facultad de Medicina, Universidad del Zulia, Maracaibo, Venezuela

2 Médico, Dr. en Ciencias Médicas, Instituto de Investigaciones Clínicas "Dr. Américo Negrette", Facultad de Medicina, Universidad del Zulia, Maracaibo, Venezuela

3 Lic. en Ciencias de la Salud y Laboratorio Clínico e Histopatológico, Instituto de Ginecología y Osteoporosis (INGINOST), Riobamba, Ecuador

4 MSc Gerencia en Salud para el Desarrollo Local, Laboratorio Clínico, Hospital General del Instituto Ecuatoriano de Seguridad Social (IESS), Riobamba, Ecuador
MSc en Bioanálisis y Diagnóstico de Laboratorio, Universidad Nacional del Chimborazo, Facultad de Ciencias de la Salud, Riobamba, Ecuador

Contacto de la autora: Adriana Pedreáñez

E-mail: apedreanez@gmail.com

Correspondencia: Apartado Postal: 23, Maracaibo 4001-A,

Zulia, Venezuela

Fecha de trabajo recibido: 25/04/21

Fecha de trabajo aceptado: 06/07/21

Conflictos de interés: los autores declaran que no existe conflicto de interés. 


\section{INTRODUCCIÓN}

La diabetes mellitus (DM) es una enfermedad metabólica caracterizada por hiperglucemia, la cual ocurre como consecuencia de defectos en la acción de la insulina sobre su receptor, en la secreción de insulina o en ambas. La hiperglucemia crónica se asocia con daño, disfunción e insuficiencia a largo plazo de diferentes órganos, especialmente ojos, riñones, nervios, corazón y vasos sanguíneos'. Es una enfermedad que ha alcanzado proporciones epidémicas. La última edición del Atlas de la Federación Internacional de Diabetes (FID) muestra que 463 millones de adultos viven actualmente con DM y se estima que habrá 578 millones de adultos con DM para el año 2030 y 700 millones para 2045. La prevalencia de DM (en adultos de 20-79 años) en América Central y América del Sur fue de 8,5\% en 2019 y se prevé que para 2030 será de 9,5 y de 9,9\% para el año $2045^{2}$.

La DM2 es la forma de diabetes más frecuente y representa entre el 90 y el $95 \%$ de las personas diagnosticadas con esta enfermedad. Anteriormente definida como DM no insulinodependiente, incluye a individuos que presentan resistencia a la insulina y usualmente deficiencia relativa (más que absoluta) de insulina?.

Los pacientes con DM suelen desarrollar diferentes complicaciones crónicas, que incluyen enfermedades macrovasculares y microvasculares ${ }^{3}$. La inflamación crónica tiene un papel importante en el desarrollo y progresión de la DM, y en la patogenia de sus complicaciones. Varios estudios informaron un incremento en los niveles de mediadores inflamatorios como la proteína $\mathrm{C}$ reactiva (PCR), la interleucina 1 (IL-1), interleucina 6 (IL-6) y el factor de necrosis tumoral alfa (TNF- $\alpha$ ) en estos pacientes ${ }^{4}$.

La proporción entre el recuento absoluto de neutrófilos y el recuento absoluto de linfocitos (índice de neutrófilos/linfocitos, INL) se ha convertido en los últimos años en un marcador crucial de inflamación sistémica ${ }^{5}$ y se ha descrito que su elevación se relaciona con numerosas enfermedades inflamatorias crónicas ${ }^{6}$.

\section{OBJETIVOS}

El objetivo de esta investigación fue determinar el INL en pacientes con DM2, comparar con individuos no diabéticos y establecer su correlación con la concentración de proteína $C$ reactiva ultrasensible (PCR-us) en una población de la localidad de Riobamba, Ecuador.

\section{MATERIALES Y MÉTODOS}

Se realizó una investigación de tipo observacional, descriptiva, correlacional, de corte transversal, en el período comprendido desde julio de 2019 a febrero de 2020.

\section{Sujetos}

El muestreo fue de tipo intencional no probabilístico. Se inscribieron sujetos que solicitaron un chequeo de rutina en un laboratorio privado de la localidad de Riobamba, Ecuador. Se seleccionaron 80 individuos para participar en el proyecto: 25 sujetos controles y 55 pacientes con diagnóstico de DM2. El diagnóstico de la DM se basó en la obtención de dos determinaciones (en dos días diferentes) de la concentración de glucosa plasmática en ayunas (después de al menos 8 horas de ayuno) utilizando un punto de corte de $126 \mathrm{mg} / \mathrm{dl}(7 \mathrm{mmol} / \mathrm{l})$.

Los criterios de exclusión fueron: evidencia de hepatitis o infección por virus de la inmunodeficiencia humana $(\mathrm{VIH}$, determinada por la presencia de anticuerpos contra los virus), manifestación de cualquier otro proceso infeccioso, inflamación activa, procesos neoplásicos malignos, y en el caso de las mujeres, la presencia de embarazo o el uso de medicamentos anticonceptivos. También se excluyeron los sujetos menores de 18 años y aquellos que estuviesen recibiendo tratamiento con esteroides.

\section{Procedimiento}

Todos los procedimientos se realizaron de acuerdo con los principios de la Declaración de Helsinki, revisada en el año 2008. El proyecto fue aprobado por la Comisión de Bioética del laboratorio involucrado y se obtuvo el consentimiento informado por escrito de todos los participantes. Se recolectaron datos como la edad, altura, peso y se calculó el índice de masa corporal (IMC) dividiendo el peso en kilogramos por el cuadrado de la altura en metros. A cada sujeto se le extrajo una muestra de sangre en ayunas para la determinación en el suero de los siguientes parámetros bioquímicos: glucosa, colesterol total, triglicéridos, HDL colesterol, LDL colesterol y PCR-us, los cuales se midieron enzimáticamente con el equipo Cobas C501 (Laboratorios Roche Diagnostics, EE.UU.), el cual se fundamenta en un método colorimétrico que utiliza un fotómetro multicanal. La hemoglobina glicosilada se evaluó por el método de Bioscience Medical SL, España, que se basa en un principio de inmunoturbidimetría. El contaje de los leucocitos y subtipos de leucocitos 
se calculó con un autoanalizador (Beckman Coulter Counter, Coulters Corporation, FL, EE.UU). El índice neutrófilos/linfocitos se definió como el recuento de neutrófilos dividido por el recuento de linfocitos.

\section{Análisis estadístico}

El análisis estadístico se realizó con el software GraphPad Instat 3.1. Los datos se expresaron como media desviación estándar (DE). La diferencia entre los grupos estudiados se evaluó mediante la prueba de Mann-Whitney. La correlación se analizó mediante la prueba de correlación de Pearson. Se consideró un valor de $p<0,05$ como estadísticamente significativo.

\section{RESULTADOS}

La población en estudio estuvo conformada por 80 sujetos: 55 pacientes con DM2 y 25 controles sanos. Se encontraron diferencias estadísticamente significativas en cuanto a edad, IMC, glucosa sanguínea, HDL colesterol y hemoglobina glicosilada al comparar los grupos estudiados (Tabla 1).

\begin{tabular}{|l|l|l|l|}
\hline \multicolumn{1}{|c|}{ Parámetros } & \multicolumn{1}{c|}{ Controles $(\mathbf{N}=\mathbf{2 5})$} & \multicolumn{1}{c|}{ Diabéticos $(\mathbf{N}=\mathbf{5 5})$} & \multicolumn{1}{c|}{ Valor de $\mathbf{p}$} \\
\hline Edad & $52,16 \pm 3,60$ & $55,36 \pm 5,97$ & $<0,01^{*}$ \\
\hline Sexo $(\%$ mujeres $)$ & $48 \%$ & $46 \%$ & NS \\
\hline Sexo $(\%$ hombres $)$ & $52 \%$ & $54 \%$ & NS \\
\hline Glucosa en ayunas $(\mathrm{mg} / \mathrm{dL})$ & $84,428 \pm 5,68$ & $130,63 \pm 20,91$ & $<0,0001^{*}$ \\
\hline HbA1c $\%)$ & $4,572 \pm 0,47$ & $7,849 \pm 0,77$ & $<0,0001^{*}$ \\
\hline IMC $\left(\mathrm{Kg} / \mathrm{m}^{2}\right)$ & $25,628 \pm 2,48$ & $29,8 \pm 2,71$ & $<0,0001^{*}$ \\
\hline Colesterol total $(\mathrm{mg} / \mathrm{dL})$ & $141,28 \pm 23,35$ & $143,13 \pm 14,80$ & $\mathrm{NS}$ \\
\hline Triglicéridos $(\mathrm{mg} / \mathrm{dL})$ & $126,84 \pm 19,53$ & $137,25 \pm 23,59$ & $\mathrm{NS}$ \\
\hline HDL colesterol $(\mathrm{mg} / \mathrm{dL})$ & $42,56 \pm 4,36$ & $40,345 \pm 2,54$ & $0,02^{*}$ \\
\hline LDL colesterol $(\mathrm{mg} / \mathrm{dL})$ & $58,96 \pm 13,87$ & $65,31 \pm 14,24$ & $\mathrm{NS}$ \\
\hline
\end{tabular}

IMC: índice de masa corporal; HbA1c: hemoglobina glicosilada; HDL= lipoproteínas de alta densidad; $L D L=$ lipoproteínas de baja densidad.

* Significancia estadística; NS= no significativo. Media desviación estándar. Prueba de Mann-Whitney.

Tabla 1: Valores de IMC y parámetros bioquímicos en pacientes con DM y controles.

Se observó una diferencia significativa entre el contaje absoluto de neutrófilos y el contaje absoluto de linfocitos entre el grupo control y los pacientes diabéticos (Tabla 2).
Con respecto al INL, se encontró un incremento significativo en los pacientes diabéticos vs los controles (control $=1,53 \pm 0,56$ vs $\mathrm{DM} 2=$ $1,90 \pm 0,52 ; p=0,0005$ ) (Figura 1).

\begin{tabular}{|l|l|l|l|}
\hline \multicolumn{1}{|c|}{ Parámetros } & \multicolumn{1}{|c|}{ Controles $(\mathbf{N}=\mathbf{2 5})$} & \multicolumn{1}{c|}{ Diabéticos $(\mathbf{N}=\mathbf{5 5})$} & \multicolumn{1}{c|}{ Valor de $\mathbf{p}$} \\
\hline $\begin{array}{l}\text { Leucocitos totales } \\
\left(10^{3} \times \mathrm{mm}^{3}\right)\end{array}$ & $6,90 \pm 0,95$ & $7,35 \pm 1,18$ & NS \\
\hline $\begin{array}{l}\text { Neutrófilos } \\
\left(10^{3} / \mu \mathrm{L}\right)\end{array}$ & $3,74 \pm 0,69$ & $4,42 \pm 0,90$ & $0,001^{*}$ \\
\hline $\begin{array}{l}\text { Linfocitos } \\
\left(10^{3} / \mathrm{\mu L}\right)\end{array}$ & $2,61 \pm 0,62$ & $2,36 \pm 0,57$ & $0,04^{*}$ \\
\hline
\end{tabular}

IMC: índice de masa corporal; HbA1c: hemoglobina glicosilada; HDL= lipoproteínas de alta densidad; $L D L=$ lipoproteínas de baja densidad.

* Significancia estadística; NS= no significativo. Media \pm desviación estándar. Prueba de Mann-Whitney.

Tabla 2: Parámetros leucocitarios en pacientes con DM y controles. 


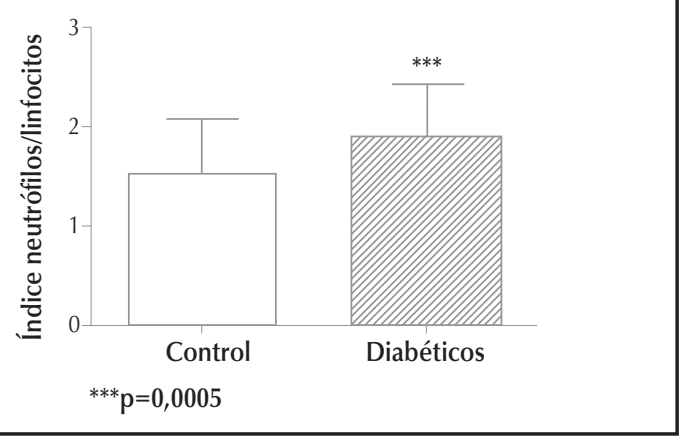

Se encontró un elevado índice neutrófilos/linfocitos en los pacientes con DM2 al compararlos con los controles. Control $(N=25)$; diabéticos ( $N=55)$. Media \pm desviación estándar. Prueba de Mann-Whitney.

Figura 1: Valores del índice neutrófilos/linfocitos.

Al evaluar la concentración de PCR-us, se observó un incremento significativo de esta molécula en los pacientes diabéticos vs controles (control $=0,39 \pm 0,15$ vs $\mathrm{DM} 2=0,65 \pm 0,27 ; \mathrm{p}<0,0001$ ) (Figura 2).

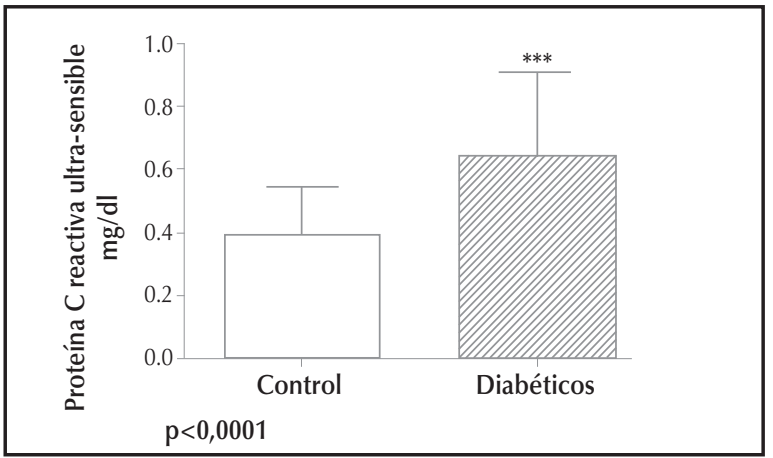

Se encontraron altos niveles de PCR-us en los pacientes con DM2 al compararlos con los controles. Control $(N=25)$; diabéticos (N=55). Media \pm desviación estándar. Prueba de Mann-Whitney.
Por otra parte, al correlacionar el índice neutrófilo/linfocitos con la concentración de PCR-us, se detectó una correlación positiva entre ambos $(p<0,0001 ; r=0,7774)$ (Figura 3 ).

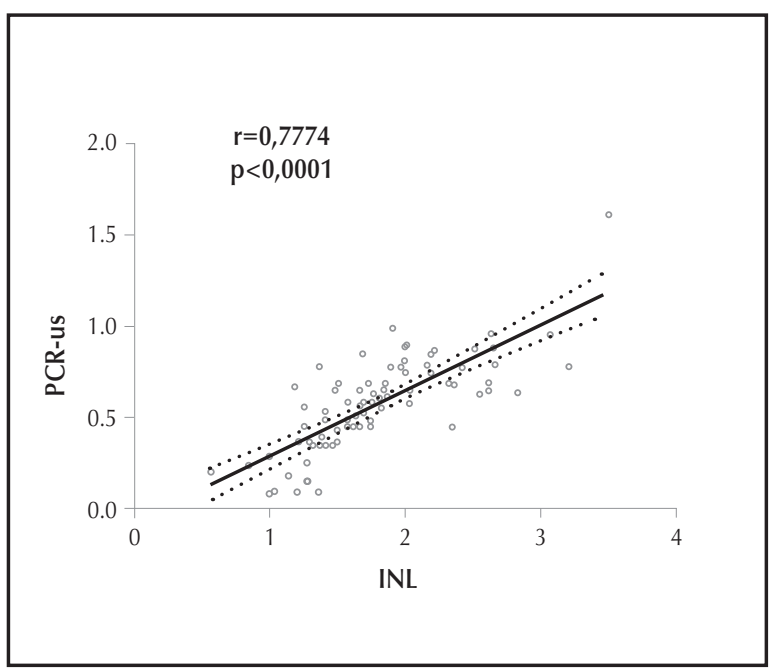

Se encontró una alta correlación positiva entre el INL y el contenido de PCR-us en los pacientes con DM2. Correlación de Pearson.

Figura 3: Correlación entre la proteína $C$ reactiva ultrasensible y el INL.

Al evaluar la correlación entre la concentración de glucosa sanguínea y el IMC con los parámetros inflamatorios estudiados se halló una correlación positiva parcial entre ellos (Tabla 3).

Figura 2: Valores de proteína $C$ reactiva ultra-sensible en suero.

\begin{tabular}{|l|l|l|}
\hline \multicolumn{1}{|c|}{ Parámetros } & \multicolumn{1}{|c|}{ Pearson (r) } & \multicolumn{1}{c|}{ Valor de $\mathbf{p}$} \\
\hline Glucemia vs PCR-us & 0,3673 & $0,0008^{*}$ \\
\hline Glucemia vs INL & 0,2829 & $0,01^{*}$ \\
\hline IMC vs PCR-us & 0,2778 & $0,01^{*}$ \\
\hline IMC vs INL & 0,1405 & NS \\
\hline
\end{tabular}

IMC = índice de masa corporal; PCR-us: proteína C reactiva ultrasensible; INL: índice de neutrófilos/linfocitos. $N S=$ no significativo estadísticamente; ${ }^{*}$ Significancia estadística.

Tabla 3: Correlación de glucemia e IMC vs parámetros inflamatorios estudiados. 


\section{DISCUSIÓN}

La DM2 es una enfermedad crónica asociada con la obesidad y la resistencia a la insulina. La aparición de esta patología está determinada principalmente por la insuficiencia progresiva de las células $\beta$ de los islotes pancreáticos para secretar niveles adecuados de insulina necesarios para mantener la normoglucemia?. Numerosos estudios han demostrado un vínculo entre la inflamación estéril de bajo grado y las enfermedades metabólicas, incluida la DM28-10.

En este sentido, se han propuesto varios marcadores biológicos que podrían indicar la gravedad de estos procesos inflamatorios. En los últimos años, el INL se ha sugerido como un indicador de inflamación subclínica y demostró ser un buen factor pronóstico para el desarrollo de complicaciones vasculares asociadas a la $\mathrm{DM}^{11-13}$. En este estudio, se evaluó el INL en una población ecuatoriana con DM2 y se encontró un incremento significativo en este marcador con respecto a los controles. Adicionalmente también se midió la concentración sérica de la proteína $C$ reactiva ultrasensible, la cual resultó igualmente elevada en estos pacientes. Estos resultados son consistentes con los reportados por otros investigadores ${ }^{14,15}$.

La DM es un trastorno metabólico asociado con un incremento de los reactantes de fase aguda. Al respecto, se ha descrito la elevación del ácido siálico, la $\alpha-1$ glicoproteína, la proteína $C$ reactiva, y otras moléculas como el amiloide sérico y la IL-6 en la $\mathrm{DM} 2^{17}$. La proteína $\mathrm{C}$ reactiva es un reactante de fase aguda sintetizada en el hígado. Su producción en los hepatocitos es inducida principalmente por la IL-6 y puede potenciarse con la IL-1 $\beta$. Es un biomarcador muy sensible, cuyos niveles séricos se elevan rápidamente en respuesta a infecciones, afecciones inflamatorias y traumatismos ${ }^{16}$. Los participantes en este estudio no presentaban alguna condición inflamatoria o enfermedad infecciosa manifiesta al momento de la toma de la muestra. Por lo tanto, la elevación en los niveles de INL y/o PCR-us en los pacientes con DM no parece ser el resultado de estas condiciones patológicas.

Es importante resaltar que los sujetos diabéticos presentaban un incremento en el IMC en comparación con los controles y pueden clasificarse como pacientes con sobrepeso (IMC: 25 a 30) ${ }^{18}$. Este estado podría contribuir a la alteración de los niveles de PCR y del INL. Al respecto, estudios previos demostraron una asociación entre el incremento del IMC y la elevación de estos parámetros ${ }^{19-22}$
Reconocemos que este aspecto es un elemento limitante de nuestros resultados ya que establecería el interrogante de si el sobrepeso en los pacientes diabéticos es un factor contribuyente al incremento de los parámetros inflamatorios estudiados. Sin embargo, como se observa en la Tabla 3, se encontró una asociación entre los valores de la PCR, el INL y los niveles de glucemia. Estos resultados no son inesperados ya que se ha descrito que la hiperglucemia en pacientes con DM2 se relaciona con el incremento en la expresión de citocinas proinflamatorias como la IL-1 $\beta$ y este fenómeno es revertido al normalizarse la concentración de glucosa sanguínea ${ }^{23}$.

La activación de mecanismos inflamatorios durante la DM posiblemente esté determinada por la estimulación del receptor para compuestos de glicación avanzada (RAGE, sus siglas en inglés)24. Nuestros resultados sugieren que la hiperglucemia se asocia más con la elevación de la PCR y el INL en los pacientes con DM2, dado que aunque el IMC se asoció al incremento de la PCR-us, no se correlacionó con la elevación del INL.

La aplicación de muchos marcadores inflamatorios se ha visto limitada en la práctica clínica diaria debido a sus costos y dificultades técnicas de medición. En esta investigación se encontró correlación positiva entre la concentración sanguínea de la PCR-us y el INL. Ambos parámetros han sido descritos como marcadores sensibles de la respuesta inflamatoria. Sin embargo, es de destacar que la medición del INL es un parámetro más económico, rutinario y fácilmente medible, calculado mediante la cuantificación de los leucocitos analizados de forma rutinaria. Por lo tanto, el INL ha sido considerado un indicador conveniente para la determinación de inflamación sistémica ${ }^{5,25}$, que refleja tanto la respuesta inmunitaria adaptativa (mediada por linfocitos) como la respuesta inmunitaria innata (mediada por neutrófilos)26,27.

Varios estudios evaluaron la importancia de la medición del INL en pacientes con DM ${ }^{28,29}$. Asimismo Mertoglu y Gunay informaron que los valores del INL eran significativamente más altos en los diabéticos recién diagnosticados en comparación con un grupo control, sugiriendo que los valores de INL pueden ser marcadores predictivos fiables en $\mathrm{DM}^{30}$. Recientemente, Wan et al. proporcionaron evidencias sobre la asociación entre el nivel del INL y las complicaciones diabéticas que incluyen la enfermedad cardiovascular (ECV), 
enfermedad renal diabética (ERD) y la retinopatía diabética (RD), en un estudio transversal que incluyó a 4.813 adultos diabéticos en siete comunidades de China. El hallazgo principal fue que el nivel de INL se asoció positivamente con ECV y $E R D$, además de RD. Hasta donde sabemos, es el primer estudio poblacional a gran escala que evaluó la asociación entre el nivel de INL y tres complicaciones vasculares crónicas en la misma población simultáneamente ${ }^{15}$.

Algunos aspectos son importantes para comprender la modificación de este parámetro en los pacientes diabéticos. En este sentido, cabe recordar que la regulación de la homeostasis del sistema inmunitario depende de la apoptosis diaria de células leucocitarias ${ }^{31}$. En este contexto, en 1948, Jailer et al. encontraron que la sobrecarga de glucosa oral tanto en diabéticos como en no diabéticos producía una disminución significativa de los linfocitos circulantes, la cual era mucho mayor en los pacientes diabéticos ${ }^{32}$. Este fenómeno fue confirmado por estudios posteriores en los cuales la tasa de apoptosis de linfocitos fue significativamente mayor en pacientes con DM2 que en la población sana ${ }^{33}$. En el presente estudio el contenido de linfocitos circulantes fue significativamente menor en los diabéticos comparado con el grupo control aunado al aumento de los neutrófilos en los pacientes diabéticos. Estos hallazgos sugieren que el incremento en el valor de INL podría resultar de la disminución en el número de linfocitos, posiblemente causada por el estrés oxidativo inducido por la hiperglucemia y la apoptosis celular.

\section{CONCLUSIONES}

En la muestra estudiada, los pacientes con DM2 mostraron una elevación en el INL que se correlacionó con un incremento en la concentración de PCR-us. Sería conveniente explorar la significación del INL como marcador de inflamación en reemplazo de la PCR-us, así como posibles puntos de corte y su valor pronóstico de complicaciones micro y macrovasculares, en estudios bien diseñados con mayor número de pacientes. El INL es un método simple y fácil de calcular. Esta prueba es económica y se realiza de forma rutinaria; puede ser un marcador alternativo efectivo del daño inflamatorio sistémico durante la DM y podría servir como sustituto de otros marcadores de inflamación como la proteína $\mathrm{C}$ reactiva.

\section{Agradecimientos}

Los autores agradecen especialmente a los pacientes que participaron en esta investigación.

\section{BIBLIOGRAFÍA}

1. American Diabetes Association. Classification and diagnosis of diabetes. Standards of Medical Care in Diabetes 2021. Diabetes Care Jan 2021; 44 (Supplement 1) S15-S33.

2. International Diabetes Federation. Revisado: 10 enero de 2021. Disponible en: http://www.idf.org/diabetesatlas.

3. Sarwar N, Gao P, Seshasai SR, Gobin R, Kaptoge S, Di Angelantonio $E$, et al. Diabetes mellitus, fasting blood glucose concentration, and risk of vascular disease: a collaborative metaanalysis of 102 prospective studies. Lancet 2010; 375:2215-2222.

4. García C, Feve B, Ferré P, Halimi S, Baizri H, Bordier L, et al. Diabetes and inflammation: fundamental aspects and clinical implications. Diabetes Metab 2010; 36:327-338.

5. Shiny A, Bibin YS, Shanthirani CS, Regin BS, Anjana RM, Balasubramanyam M, et al. Association of neutrophil-lymphocyte ratio with glucose intolerance: an indicator of systemic inflammation in patients with type 2 diabetes. Diabetes TechnolTher 2014; 16:524-530.

6. Balta S, CelikT, Mikhailidis DP, Ozturk C, Demirkol S, Aparci M, et al. The relation between atherosclerosis and the neutrophillymphocyte ratio. Clin ApplThromb Hemost 2016; 22:405-411.

7. Donath MY, Boni-Schnetzler M, Ellingsgaard H, Ehses JA. Islet inflammation impairs the pancreatic beta-cell in type 2 diabetes. Physiology (Bethesda) 2009; 24:325-331.

8. Donath MY, Shoelson SE. Type 2 diabetes as an inflammatory disease. Nat Rev Immunol 2011; 11:98-107.

9. Larsen CM, Faulenbach M, Vaag A, Volund A, Ehses JA, Seifert $B$, et al. Interleukin-1-receptor antagonist in type 2 diabetes mellitus. N Engl J Med 2007; 356:1517-1526.

10. Esser N, Legrand-Poels S, Piette J, Scheen AJ, Paquot N. Inflammation as a link between obesity, metabolic syndrome and type 2 diabetes. Diabetes Res Clin Pract 2014; 105:141-150.

11. DiGangi C. Neutrophil-lymphocyte ratio: predicting cardiovascular and renal complications in patients with diabetes. J Am Assoc Nurse Pract 2016; 28:410-414.

12. Wang J, Zhu OW, Cheng $X Y$, Sha CX, Cui YB. Clinical significance of neutrophil-lymphocyte ratio and monocyte-lymphocyte ratio in women with hyperglycemia. Postgrad Med 2020; 132:702-708.

13. Li J, Li T, Wang H, Yan W, MuY. Neutrophil-lymphocyte ratio as a predictor of kidney function decline among individuals with diabetes and prediabetes: A 3-year follow-up study. J Diabetes 2019; 11:427-430.

14. Verdoia M, Schaffer A, Barbieri L, Aimaretti G, Marino P, Sinigaglia $\mathrm{F}$, et al. Impact of diabetes on neutrophil-to-lymphocyte ratio and its relationship to coronary artery disease. Diabetes Metab 2015; 41:304-311.

15. Wan $H$, Wang $Y$, Fang $S$, Chen $Y$, Zhang W, Xia F, et al. Associations between the neutrophil-to-lymphocyte ratio and diabetic complications in adults with diabetes. A cross-sectional study. J Diabetes Res 2020; 1-9.

16. Black S, Kushner I, Samols D. C-reactive protein. J Biol Chem 2004; 279:48487-48490.

17. Pitsavos C, Tampourlou M, Panagiotakos DB, Skoumas Y, Chrysohoou C, Nomikos T, et al. Association between low-grade systemic inflammation and type 2 diabetes mellitus among men and qomen from the ATTICA Study. Rev Diabet Stud 2007; 4:98-104.

18. Shiwaku K, Anuurad E, Enkhmaa B, Nogi A, Kitajima K, Shimono $\mathrm{K}$, et al. Overweight Japanese with body mass indexes of 23.024.9 have higher risks for obesity-associated disorders: a comparison of Japanese and Mongolians. International Journal of Obesity and Related Metabolic Disorders 2004; 28 (1):152-158. 
19. Ryder E, Pedreañez A, Vargas R, Peña C, Fernández E, DiezEwald $M$, et al. Increased proinflammatory markers and lipoperoxidation in obese individuals. Inicial inflammatory events? Diabetes Metab Syndr 2015 Oct-Dec; 9(4):280-6.

20. Ryder E, Diez-Ewald M, Mosquera J, Fernández E, Pedreañez A, Vargas $R$, et al. Association of obesity with leukocyte count in obese individuals without metabolic syndrome. Diabetes Metab Syndr 2014 Oct-Dec; 8(4):197-204.

21. Vargas R, Ryder E, Diez-Ewald M, Mosquera J, Durán A, Valero $\mathrm{N}$, et al. Increased C-reactive protein and decreased interleukin-2 content in serum from obese individuals with or without insulin resistance. Associations with leukocyte count and insulin and adiponectin content. Diabetes Metab Syndr 2016 JanMar; 10(1 Suppl 1):S34-41.

22. Yilmaz $H$, Ucan B, Sayki M, Unsal I, Sahin M, Ozbek M, et al. Usefulness of the neutrophil-to-lymphocyte ratio to prediction of type 2 diabetes mellitus in morbid obesity. Diabetes Metab Syndr 2015 Oct-Dec; 9(4):299-304.

23. Iglesias-Molli $A E$, Bergonzi MF, Spalvieri MP, Linari MA, Frechtel GD, Cerrone GE. Relationship between the IL-1 $\beta$ serum concentration, mRNA levels and rs16944 genotype in the hyperglycemic normalization of T2D patients. Sci Rep 2020 Jun 19; 10(1):9985.

24. Byun K, YooY, Son M, Lee J, Jeong GB, ParkYM, Salekdeh GH, Lee B. Advanced glycation end-products produced systemically and by macrophages: A common contributor to inflammation and degenerative diseases. Pharmacol Ther 2017 Sep; 177:44-55.

25. Gibson $\mathrm{PH}$, Cuthbertson $B \mathrm{H}$, Croal BL, Rae D, El-Shafei $H$, Gibson $\mathrm{G}$, et al. Usefulness of neutrophil/lymphocyte ratio as predictor of new-onset atrial fibrillation after coronary artery bypass grafting. Am J Cardiol 2010; 105:186-191.
26. Sawant AC, Adhikari P, Narra SR, Srivatsa SS, Mills PK, Srivatsa SS. Neutrophil to lymphocyte ratio predicts short- and long-term mortality following revascularization therapy for ST elevation myocardial infarction. Cardiology Journal 2014; 21:500-508.

27. Tamhane UU, Aneja S, Montgomery D, Rogers EK, Eagle KA Gurm HS. Association between admission neutrophil to lymphocyte ratio and outcomes in patients with acute coronary syndrome. The American Journal of Cardiology 2008;102:653-657.

28. Azab B, Chainani V, Shah N, McGinn JT. Neutrophil-lymphocyte ratio as a predictor of major adverse cardiac events among diabetic population: a 4-year follow-up study. Angiology 2013; 64:456-465

29. Azab B, Daoud J, Naeem FB, Nasr R, Ross J, Ghimire P, et al. Neutrophil-to-lymphocyte ratio as a predictor of worsening renal function in diabetic patients (3-year follow-up study). Ren Fail 2012; 34:571-576.

30. Mertoglu C, Gunay M. Neutrophil-lymphocyte ratio and plateletlymphocyte ratio as useful predictive markers of prediabetes and diabetes mellitus. Diabetes Metab Syndr 2016; 11:127-131.

31. Cohen JJ, Duke RC, Fadok VA, Sellins KS. Apoptosis and programmed cell death in immunity. Annu Rev Immunol 1992; 10:267-293.

32. Jailer JW, Marks DT, Marks PA. Variations in white blood cells following the oral administration of glucose to diabetics and nondiabetics. J Clin Endocrinol Metab 1948; 8:1074-1080.

33. Xu H, Chen Y, Li Y, Xia F, Han B, Zhang $\mathrm{H}$, et al. Mitochondrial apoptosis of lymphocyte is induced in type 2 diabetes. Chin Med J (Engl) 2014; 127:213-217. 